\title{
Type IIn SN 2010jl: probing dusty line-emitting shell
}

\author{
Nikolai N. Chugai, ${ }^{1 \star}$ \\ Institute of Astronomy Russian Academy of Science, Moscow, Russia
}

Accepted XXX. Received YYY; in original form ZZZ

\begin{abstract}
I explore signatures of a possible dust formation in the late SN 2010jl that could be imprinted in the line blueshift and the radius evolution of the dusty infraredemitting shell. I propose a simple model that permits one to reproduce emission lines of blueshifted hydrogen and helium emission lines. The model suggests that the hydrogen emission originates primarily from shocked fragmented circumstellar clumps partially obscured by the absorbing cool dense shell and by unshocked ejecta. In the He $1.083 \mu \mathrm{m}$ line on day 178 this component is significantly weaker compared to broad component from unshocked ejecta that is obscured by the absorprion produced by ejecta itself. Simulations of late time $(t>400 \mathrm{~d}) \mathrm{H} \alpha$ suggest that, apart from the dust in the cool dense shell, a significant amount of dust must form in the unshocked supernova ejecta. The supernova radius predicted by the interaction model coincides with the radius of the dusty shell recovered from late time (> 460 days) infrared data, which strongly support that infrared radiation indeed originates from supernova. The ejecta dust is presumably locked in opaque blobs.
\end{abstract}

Key words: supernovae - individual - SN 2010j1

\section{INTRODUCTION}

The luminous supernova SN 2010jl interacting with a dense circumstellar matter (CSM) is a special case among SNe IIn due to a large volume of observational data accumulated in different bands including X-ray (Chandra et al. 2012; Ofek et al. 2014; Chandra et al. 2015), optical (Stoll et al. 2011; Zhang et al. 2012; Smith et al. 2012; Ofek et al. 2014; Fransson et al. 2014; Gall et al. 2014; Borish et al. 2015; Jencson et al. 2016), infrared (IR) (Andrews et al. 2011; Fransson et al. 2014; Gall et al. 2014; Borish et al. 2015; Dwek et al. 2017), and radio (Chandra et al. 2015). This provide us with an opportunity to get deeper insight into complicated phenomena accompanied the ejecta interaction with a dense CSM. Of particular interest is the suspected dust formation in the postshock cool dense shell (CDS) indicated by the late time hot IR excess (Maeda et al. 2013; Gall et al. 2014) combined with the line blueshift (Smith et al. 2012). The estimated amount of the dust is of $\approx 10^{-3} M_{\odot}$ after about 500 d (Maeda et al. 2013; Gall et al. 2014; Sarangi et al. 2018).

The possibility of the dust formation in the CDS of SNe IIn was discussed earlier for SN 1998S (Pozzo et al. 2004), SN 2006jc (Smith et al. 2008; Mattila et al. 2008; Chugai 2009), SN 2005ip (Smith et al. 2009), SN 2007od (Andrews et al. 2010). Yet there are open questions that

^ E-mail: nchugai@inasan.ru may cast doubts on this conjecture. Among them is the issue of so called blueshifted Lorentz-like profiles (narrow core plus broad wings). The point is that, if the dusty lineemitting shell is composed of the CDS material only, one would expect then to find a blueshifted broad boxy line profile because in this case the velocity dispersion of the lineemitting material is small compared to the expansion velocity. This issue was emphasised by Fransson et al. (2014) to argue in favour of the CS dust. The concept of the external dust however leaves blueshifted Lorentz-like profiles unexplained. Since the conjecture on the dusty CDS and the line blueshift seems to be closely linked the origin of the Lorentzlike profile requires an explanation before a confident conclusion on the dust location could be made. Furthermore, accepting the idea of the dust formation in the CDS we have not so far answers to a straightforward questions concerning the site and velocity distribution of the line-emitting gas and the dust distribution. It is noteworthy that, apart from the CDS, the dust in SNe IIn might form also in unshocked ejecta in the same way as it does in SNe IIP, e.g., SN 1987A (Lucy et al. 1989) and SN 1999em (Elmhamdi et al. 2003). Note, the dust formation in the unshocked ejecta of SN IIn has been already proposed in a context of an infrared excess in the SN 2005ip (Fox et al. 2010). This possibility should be considered as highly probable for SN 2010jl as well.

Below I address two principal questions: (i) what is the origin of blueshifted lines in spectra of SN 2010jl; (ii) whether the late time $\mathrm{SN}$ radius is consistent with the radius 
of the IR-emitting dust shell. The answer to these questions will permit us to present at least a qualitative model that accounts for blueshifted line profiles and late IR emission in unique picture lacking at the moment. I start with an explantion of the origin of Lorentz profile and the blueshift modelling (Section 2). This is followed by the modelling of the SN/CSM interaction that provides us with an important tool for the probing the relation between the $\mathrm{SN}$ radius and the radius of the IR-emitting shell (Section 3). In the Discussion section the clumpy dust distribution is discussed and the estimate of the dust amount is given.

Following Fransson et al. (2014) we adopt the explosion date 2010 October 9 (JD 2455479).

\section{SPECTRAL MODELLING}

\subsection{Preliminaries: Why Lorentz profile?}

The interaction of spherically-symmetric freely expanding $(v=r / t)$ SN ejecta with a spherically-symmetric CSM occurs via the formation of two shock waves: forward (radius $R_{f}$ ) and reverse shock (radius $R_{r}$ ) with a shocked SN and CS matter swept-up in a thin dense shell at the contact surface (radius $R_{0}$ ). (Henceforth radii are measured in units of $R_{0}=1$, if otherwise not stated explicitly). In an adiabatic case for the typical density distribution of ejecta $\left(\rho \sim v^{-8}\right)$ and CSM $\left(\rho \sim r^{-2}\right)$ the shock radii ratios are $R_{r} / R_{0}=0.98$ and $R_{f} / R_{0}=1.27$ (Chevalier 1982a). In strongly radiative regime the postshock layers get narrower, so one can adopt in this case $R_{r}=R_{0}$. The X-rays from both shocks excite cold unshocked SN ejecta and CSM thus giving rise to optical broad lines and narrow lines respectively. In a realistic situation the picture grow significantly more complicated: the flow between forward and reverse shock turns out three-dimensional due to Rayleigh-Taylor (RT) instability of the thin shell (Chevalier 1982b; Chevalier \& Blondin 1995; Blondin \& Ellison 2001) and because of a lumpiness of the CSM. Therefore, in reality additional line-emitting sites related to the CDS and shocked CS clouds can appear between forward and reverse shock. Due to the non-trivial velocity spectrum and highly complicated radiation transfer effects the optical radiation spectrum of SNe IIn cannot be reliably predicted so far.

Emission lines of SNe IIn at the early stage $(t<1 \mathrm{yr})$ usually (but not always) reveal three components: the narrow component (NC), broad (BC), and intermediate (IC), all originally have been recognized in the SN $1988 \mathrm{Z}$ spectrum by Filippenko (1991). These components can be identified (Figure 1) with the CSM, unshocked SN ejecta in combination with the CDS, and shocked CS clouds, respectively, (Chugai \& Danziger 1994). In SN 2010jl the distinction between BC and IC is not so apparent as in SN 1988Z: hydrogen lines, e.g. $\mathrm{H} \alpha$, of SN 2010jl are rather smooth and indeed reminds a "Lorentz" profile (Fransson et al. 2014). We will use this term, keeping in mind that it has nothing to do with the Lorentz distribution of frequencies of the damping oscillator radiation. Fortunately, in He I $1.083 \mu \mathrm{m}$ line the component ratio of $\mathrm{BC} / \mathrm{IC}$ is large, so both components are easily distinguished (Borish et al. 2015), which keeps safe canonical three-component structure of emission lines for SN 2010jl.
It should be emphasised that late time Lorentz profiles in SN 2010jl have essentially different origin than emission lines with Lorentz profile in early spectra of some SNe IIn, identified with emission of the ionized dense preshock CSM, where these lines get broadened by the Thomson scattering; the phenomenon recognized for SN 1998S (Chugai 2001). This type of the profile cannot present at late epochs $(t \gtrsim 100 \mathrm{~d})$ since the required Thomson optical depth of a preshock CSM must be large $\left(\tau_{\mathrm{T}} \gtrsim 2\right)$ that is unattainable at that late time.

The Lorentz profile in the late time SNe 2010jl can be interpreted following a suggestion invoked for SN 2006jc (type IIn), where the emission lines have been attributed to shocked fragmented CS clouds (Chugai 2009). A typical value of the cloud shock in SNe IIn is $v_{c} \sim 10^{3} \mathrm{~km} \mathrm{~s}^{-1}$ (e.g., SN 1988Z, Chugai \& Danziger 1994), which for the postshock intercloud velocity $v_{i c} \approx 6000 \mathrm{~km} \mathrm{~s}^{-1}$ implies the CS cloud density contrast with respect to the intercloud density $\chi \approx\left(v_{i c} / v_{c}\right)^{2} \sim 30-40$. The shocked fragmented CS clouds resides between the forward shock and the CDS (Figure 1) while their velocities range between the initial cloud shock velocity $\left(v_{c}\right)$ and the final velocity of fragments $v_{\max } \approx(0.75 \ldots 0.9) v_{i c}$ for $10<\chi<100$ (Klein et al. 1994). Note, the velocity the postshock intecloud gas coincides with the CDS velocity $v_{c d s}$. The cloud fragmentation and fragments acceleration by the incident shock is well demonstrated by laser experiments and 3D-hydrodynamic simulations Klein et al. (2003) with the relevant physics well understood earlier (Klein et al. 1994).

The case of SN 2006jc indicates that the Lorentz profiles can be reproduced with the velocity distribution of the line emissivity $j(v) \propto\left(v_{\max }-v\right)$ (Chugai 2009). The following toy model illustrates, how such a velocity spectrum could arise. Consider a steady-state flow of CS clouds into the forward shock with the rate $G$ (clouds/s). The cloud in the forward shock experiences crushing by the radiative shock followed by the fragmentation, acceleration of fragments, and fragments mixing with ambient intercloud hot gas (Klein et al. 1994). Let cloud life span in the forward shock be $T$ and the cloud survival probability at the age $\tau$ be the linear function of the age $p(\tau) \propto(1-\tau / T)$. Assuming that the fragmented cloud accelerates linearly as $v=v_{c}+a \tau$, which is close to 2D-simulation results (Klein et al. 1994), and taking into account that the clouds age distribution is $d N / d \tau=G p(\tau)$ one finds the velocity distribution of shocked clouds $d N / d v=(d N / d \tau)(d \tau / d v) \propto\left(v_{\max }-v\right)$, where $v_{\max }=v_{c}+a T$. With the constant specific emissivity (per gramm) we thus come to the required velocity distribution of the line emissivity. This is only crude illustration and the emissivity velocity distribution may difer from this simple law.

\subsection{Line profile}

\subsubsection{Model}

A minimal model that captures major structure elements involved in the line profile formation of SN 2010jl includes three components: (i) freely expanding SN ejecta $(v=r / t$, $\left.r<R_{0}=1\right)$; (ii) perturbed CDS $\left(R_{0}<r<R_{1}=1.1\right)$ with the average velocity $v_{c d s}$ and a random component in the range of $\delta v \sim 0.1 v$ (e.g., Blondin \& Ellison 2001); (iii) line-emitting 


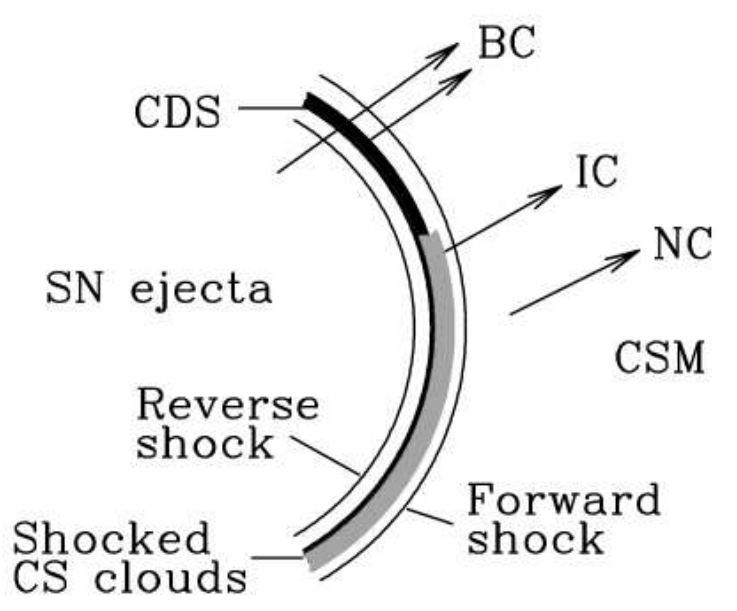

Figure 1. Schematic diagram of the SN/CSM interaction showing source location for the narrow, broad and intermediate components involved in the overall line emission. The layer of cold CDS material is partially mixed with the layer of shocked CS clouds (grey); only a fragment of the latter is shown for clarity.

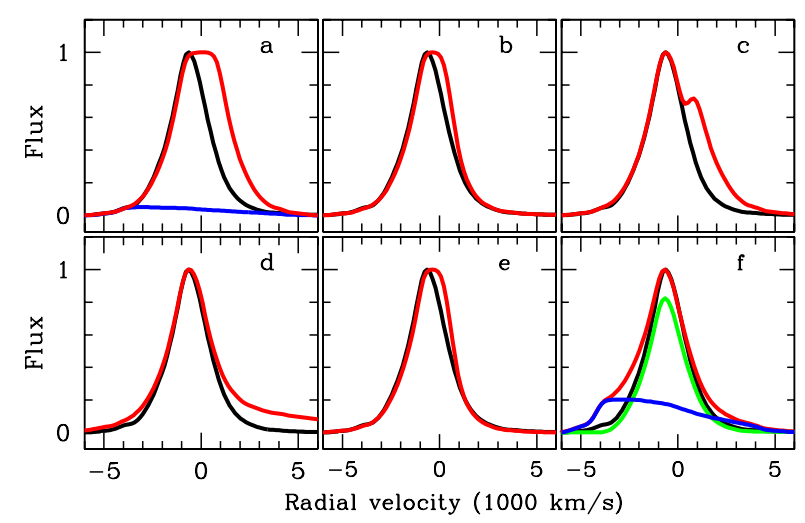

Figure 2. Effects of the parameters variation on the $\mathrm{H} \alpha$ model profile. Panels from a to $\mathbf{f}$ show the fiducial model mod0 (black) and models by order from mod1 to mod6 with changed parameter value (red) (Table 2). In the panel a the combined contribution of broad components of the CDS and ejecta in the mod0 is shown for clarity (blue). The panel $\mathbf{f}$ demonstrates effect of a large contribution of the CDS and unshocked ejecta emission (blue) while the intermediate component (green) remains unchanged.

Table 1. Parameters of demonstration models.

\begin{tabular}{ccccccc}
\hline Model & $\tau_{c d s}$ & $\tau_{s n}$ & $\omega_{s n}$ & $R_{2}$ & $f_{c d s}$ & $f_{s n}$ \\
\hline $\bmod 0$ & 0.13 & 1 & 0.1 & 1.1 & 0.05 & 0.08 \\
$\bmod 1$ & 0 & 0 & 0.1 & 1.1 & 0.05 & 0.08 \\
$\bmod 2$ & 0 & 1 & 0.1 & 1.1 & 0.05 & 0.08 \\
$\bmod 3$ & 0.13 & 0 & 0.1 & 1.1 & 0.05 & 0.08 \\
$\bmod 4$ & 1.13 & 1 & 1 & 1.1 & 0.05 & 0.08 \\
$\bmod 5$ & 0.13 & 1 & 0.1 & 1.2 & 0.05 & 0.08 \\
$\bmod 6$ & 0.13 & 1 & 0.1 & 1.1 & 0.2 & 0.2 \\
\hline
\end{tabular}

fragments of shocked CS clouds $\left(R_{0}<r<R_{2}\right)$ macroscopically mixed with the absorbing CDS material. This component is identified with the line intermediate component, while former two are responsible for the broad component. The narrow component originated in the preshock CSM is not considered in our model. I neglect the hot thin layer in the reverse shock and set the radius of the unshocked SN ejecta equal to the CDS radius $R_{0}$.

In line with arguments of Section 2.1 the emissivity velocity distribution for the shocked CS clouds responsible for the intermediate component is approximated by the function

$$
g(v)=\left\{\begin{array}{cc}
\frac{v-v_{\min }}{v_{c}-v_{\min }}, & \text { if } v_{\min }<v<v_{c} \\
{\left[\frac{v_{\max }-v}{v_{\max }-v_{c}}\right]^{q},} & \text { if } v_{c}<v<v_{\max } \\
0, & \text { otherwise }
\end{array}\right.
$$

where the index $q$ is a free parameter close to unity. The function $g(v)$ has a maximum at $v=v_{c}$ with the linear rise in the range $v_{c, \text { min }}<v<v_{c}$ that makes allowance for the fact that CS clouds are not identical; we adopt $v_{\text {min }}=0.9 v_{c}$. Note that in the case of $v_{\text {min }}=v_{c}$ the line profile would be flat in the range of radial velocities $\pm v_{c}$ which is not seen in observed profiles. I preliminary explored two radial distribution of the emissivity of shocked clouds: (i) uniform in the range $R_{0}<r<R_{2}$ (i.e., no radius/velocity correlation) and (ii) linear radius/velocity correlation, viz. $r=R_{0}+\left(R_{2}-\right.$ $\left.R_{0}\right)\left(v-v_{\text {min }}\right) /\left(v_{\max }-v_{\text {min }}\right)$. The resulting profiles in these cases are indistiguishable, except for the late spectrum on day 804 when the correlation produces slightly better fit. This choice therefore is applied for all the epochs.

The broad component is emitted by the CDS and unshocked ejecta with relative fractions $f_{c d s}$ and $f_{s n}$, so the intermediate component fraction is $1-f_{c d s}-f_{s n}$. The CDS emissivity is distributed homogeneously in the velocity randomly distributed in the range of $v_{c d s}<v<1.1 v_{c d s}$. The emissivity distribution in the SN ejecta is described by the "broken" power law $j(v)=\left(v / v_{b}\right)^{k_{1}} /\left[1+\left(v / v_{b}\right)^{k_{2}}\right]$ with the velocity turnover $v_{b}$ and indices $k_{1} \approx 2$ and $k_{2} \approx 9$ inferred from broad component of the He I $1.083 \mu \mathrm{m}$ line. The turnover of the $j(v)$ function is expected because the ionizing radiation from the shocks is strongly absorbed in outer layers of ejecta.

The optical line emitted by ejecta can be scattered by electrons and dust (if any) and absorbed by the hydrogen and dust. The distribution of absorbers in SN ejecta and CDS is assumed to be homogeneous along the radius. The absorption is specified by the optical depth of the CDS and ejecta $\left(\tau_{c d s}\right.$ and $\left.\tau_{s n}\right)$, and albedo $(\omega)$, viz. the ratio of the scattering to extinction coefficient. To reduce the number of free parameters the albedo is set to be the same in the CDS and ejecta, which may not be the case. However this assumption does not affect significantly results because, as we will see, the ejecta dominates in the observable scattering effect. In search for the optimal optical depth and albedo I ignore a possible lumpiness of the dust distribution. However, since effects of clumpy dust distribution will be discussed below, it is instructive to make some remarks concerning this issue.

The effective optical depth in the clumpy medium is introduced Ambarzumian (1947) as $\tau_{e f f}=\tau_{o c}\langle 1-\exp (-\tau)\rangle$, where $\tau_{o c}$ is the number of clouds along the ray, or the occultation optical depth, $\tau$ is the random cloud optical depth, and the averaging should be performed over cloud cross sec- 

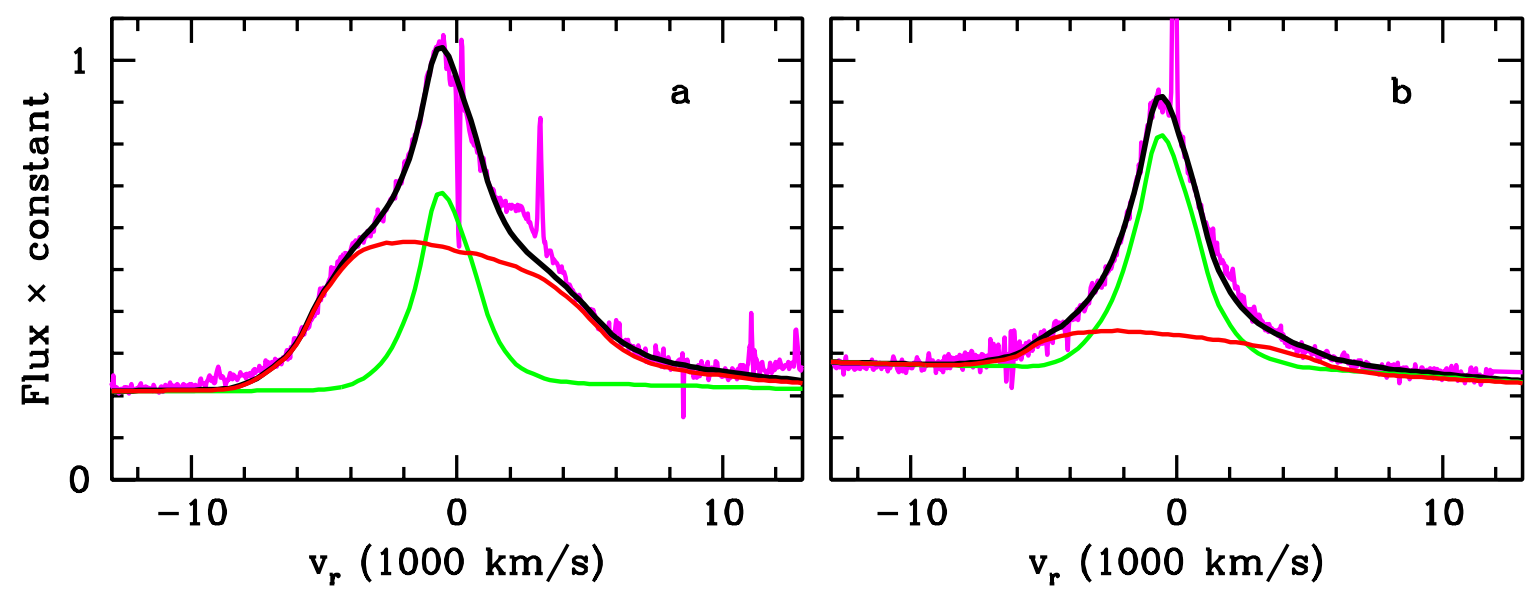

Figure 3. The HeI $1.083 \mu \mathrm{m}$ (panel a) and $\mathrm{Pa} \beta$ (panel b) in SN 2010jl on day 178 (magenta) compared to models. The excess in the red wing of the HeI $1.083 \mu \mathrm{m}$ is due to Pa . Shown also contributions of the intermediate component (green line) and combined broad components of CDS and unshocked ejecta $(r e d)$.

tion and cloud ensemble. In the toy model of homogeneous spherical clouds with of the radius $b$ and the optical radius $\tau=k b$ homogeneously distributed in a sphere of a radius $R$ the average is calculated analytically (Utrobin \& Chugai $2015)$ and reads $\langle\ldots\rangle=(4 \tau / 3) p(\tau)$, where $p(\tau)$ is the escape probability of photon emitted in the homogeneous sphere of the optical radius $\tau$ (see Osterbrock 1989). In the limit of $\tau \ll 1$ retaining only linear terms we get $\tau_{\text {eff }}=(4 / 3) \tau \tau_{o c}$ that can be easily transformed into $\tau_{\text {eff }}=f k R$, where $f$ is the filling factor of clouds. In the oposite case of $\tau \gg 1$ we obtain $\tau_{\text {eff }}=\tau_{o c}$ in line with the euristic definition of the optical depth for the ensemble of clouds treated as macroatoms.

The optical depth recovered in the homogeneous model in clumpy case should be identified with the effective optical depth $\tau_{e f f}$ and in the case of optically thick clouds this will be simply $\tau_{o c}$. The albedo of the homogeneous model $(\omega)$ for the clumpy medium should be associated with the albedo of a dusty cloud, which is expected to be lower than single scattering albedo of a grain $\left(\omega_{d}\right)$. The relation between $\omega_{d}$ and $\omega$ can be illustrated by Monte Carlo simulations for the spherical dusty cloud and spherical phase function. In the limit of a large cloud optical radius $(\tau>10)$ for the set of the single scattering albedo $(0.1,0.3,0.6,0.9)$ the asymptotic values of $\omega$ turn out to be $0.023,0.08,0.2$, and 0.5 , respectively. If, e.g., one finds in the homogeneous model $\omega=0.1$, then in the clumpy case with the large optical depth of the dusty cloud one expects the single scattering albedo to be $\omega_{d} \approx 0.35$.

The apparent drawback of the outlined model is a large number of free parameters even in a homogeneous case. The list includes $R_{2}, v_{s n}, v_{c d s}, v_{c}, v_{\max }, q, \tau_{c d s}, \tau_{s n}, \omega, f_{c d s}$ and $f_{s n}$, among which four parameters $\tau_{c d s}, \tau_{s n}, \omega$, and $v_{s n}$ are of prime interest for us. The rest of parameters are constrained with different precisions. Particularly, at late time the contribution of the CDS is poorly constrained, so neither $f_{c d s}$ and $v_{c d s}$ can reliably inferred. Fortunately this does not affect the determination of the principal four parameters. Although an effect of each parameter can be controlled, the problem of the unique set of parameters that defines the best fit model in multi-dimensional parameter space would be unproductive to solve rigorously, because the model is rather crude and ignores a possible deviation from the spherical symmetry that is plausible given a significant intrinsic polarization found in type IIn SN 1997eg (Hoffman et al. 2008), in some aspects reminding SN 2010jl.

\subsubsection{Results}

In a search for the optimal model I explored an extended volume of parameter space. Fortunately effects of most parameters are rather obvious, although in some cases the effect is less trivial. To provide insight into the role of principal paprameters I present a set of simulated Lorentz-like profiles (Table 1, Fig. 2). Each panel shows a template model (mod0) that corresponds to the $\mathrm{H} \alpha$ model on day 510 and a model with the altered parameter value. The template model is dominated by the emission of shocked CS clouds (intermediate component); ejecta and CDS contribute together $10 \%$ (panel a). The unabsorbed line with zero optical depth for both CDS and SN ejecta (panel a) shows symmetriic profile with roundish top that is due to the low velocity increasing part of the velocity spectrum $g(v)$ of shocked CS clouds (cf. Section 2.2.1). The case of a zero CDS absorption (panel b) demonstrates that the ejecta absorption is not sufficient to produce required blueshift of the line maximum. On the other hand, the absence of the ejecta absorption (panel c) shows that while the CDS absorption is able to describe the blueshift of the maximum, the profile in the red is unlike what is required. Note that the dip in the red part is a limb effect for the line arising in a narrow shell $(\Delta R / R<1)$ with the continuum absorption/scattering (cf. Chugai 1991). The case of $\omega=1$ corresponding to the Thomson scattering with the thermal broadening taken inro account results in the pronounced red wing (panel d), a natural outcome of the photon scattering in the expanding envelope (Auer \& van Blerkom 1972). This effect is also present in the case when line photons are scattered off a dust with albedo $\omega \sim 0.5$ in SN ejecta (Lucy et al. 1989; Bevan \& Barlow 2016). The need for the macroscopic mixing between the layer of shocked CS clouds 
and the dusty CDS material is demonstrated by model with the outward shift of the line-emitting layer by $\Delta r=0.1 R_{0}$ (panel e): the model shows lower maximum blueshift. Interestingly, the profile in the model mod5 almost exactly repeats the profile in the model $\bmod 2$. This is not unexpected since in both cases line-emitting shell lies above the absorbing shell. This similarity however cannot be considered as the parameter degeneracy, because in the model with correct blueshift these similar cases cannot appear. The effect of large contribution of the broad components related to CDS and SN ejecta indicates how the broad components can be constrained (panel $\mathbf{f}$ ). The value of the index $q$ affects the intermediate component in a sense that $q \gg 1$ makes profile narrower and more gaussian-like, while $q \ll 1$ makes profile broader and closer to the triangular shape.

We now consider an interesting case of the HeI 1.083 $\mu \mathrm{m}$ line. At the early stage $(t<400 \mathrm{~d})$ He I $1.083 \mu \mathrm{m}$ and HeI $2.06 \mu$ m lines show a pronounced broad component unlike smooth hydrogen line profiles (Borish et al. 2015). A reasonable suggestion is that this component is caused by the emission of the SN ejecta (Borish et al. 2015). Figure 3 demonstrates satisfactory fits HeI $1.083 \mu \mathrm{m}$ and $\mathrm{Pa} \beta$ on day 178 in the models with the same parameters except for the ejecta emission fraction, $f_{s n}=0.68$ for HeI $1.083 \mu \mathrm{m}$ compared to 0.3 for for $\mathrm{Pa} \beta$, and the index, $q=1.5$ for He I $1.083 \mu \mathrm{m}$ compared to $q=0.9$ for $\mathrm{Pa} \beta$. The latter difference presumably reflects different excitation conditions of hydrogen and helium in the intermediate component. Both helium and hydrogen line shows blueshift that is expressed in the inferred values of optical depths $\tau_{c d s}=0.07, \tau_{s n}=0.58$, and albedo $\omega=0.6$. Since at this epoch the dust formation in the CDS and SN ejecta is unlikely, the extiction is probaly related to the hydrogen photoabsorption and Thomson scattering. It should be emphasised that the blueshift of the broad component of HeI $1.083 \mu \mathrm{m}$ is produced entirely by the absorption in the unshocked ejecta. It is not clear whether the different fraction of the broad component for helium and hydrogen lines is related to the different helium abundance in SN ejecta and CSM, or different excitation conditions in the line-emitting regions. Discussion of this issue would require highly complicated modelling, which is currently beyond reach. The recovered parameters of the HeI ejecta component, viz. $v_{b}=5500 \mathrm{~km} \mathrm{~s}^{-1}, k_{1}=2$, and $k_{2}=9$ (cf. Equation 1) are adopted henceforth for hydrogen lines as well because in hydrogen lines the ejecta component parameters cannot be reliably constrained.

The $\mathrm{H} \alpha$ line is modelled for spectra taken on 2011 March 31 (172 days), 2011 Nov. 13 (400 days), 2012 Feb. 28 (510 days), and 2012 Dec. 21 (804 days). The data are retrieved from the Weizmann supernova data repository http://wiserep.weizmann.ac.il (Yaron \& Gal-Yam 2012). Models overplotted on the observed spectra are displayed in Fig. 4 with parameters given in the Table 2. Columns one by one contain: the model name (the number is the age in days), terminal velocity of unshocked SN ejecta $\left(v_{s n}\right)$, CDS velocity $\left(v_{c d s}\right)$, velocity of CS cloud shock $\left(v_{c}\right)$, maximal velocity of the CS shocked cloud material $\left(v_{\max }\right)$, index $q$ of the emissivity of shocked CS clouds, outer radius of the line-emitting layer of shocked CS clouds $\left(R_{2}\right)$; continuum optical depths of the CDS $\left(\tau_{c d s}\right)$ and unshocked SN ejecta $\left(\tau_{s n}\right)$; albedo $(\omega)$ and the relative contribution of the CDS $\left(f_{c d s}\right)$ and unshocked SN $\left(f_{s n}\right)$ into the $\mathrm{H} \alpha$ luminosity. The values in parentheses have large uncertainties. For all the considered epochs the CDS contribution is dominant. The contribution of the broad components both ejecta and the CDS is large only in the spectrum on day 172 and gets small with the age.

Remarkably, the CDS optical depth at the late stage (510 and $804 \mathrm{~d}$ ), when presumably the dust have been formed, is rather small ( 0.13 and 0.15 respectively), whereas SN ejecta turns out essentially more opaque $\left(\tau_{s n}=1\right)$. This indicates that apart from the CDS the dust also forms in the unshocked ejecta and possibly in larger amount. Note that at late time the lower albedo is preferred. This fact is consistent with the dust to be the main source of opacity, since the hydrogen density and hydrogen excitation in the SN ejecta decreses with time and therefore the hydrogen absorption and Thomson scattering gets inefficient.

The estimated parameter errors that reflect precision of model fits lie in the range 10-20\% except for those inicated by parentheses in Table 2. The errors however reflect only the adopted model. In fact, the radial distribution of absorbers and emitters in ejecta and velocity and radial distributions of shocked CS clouds might be more complicated; moreover, the anisotropy is not rulled out. All these can result in some change of values of four principal parameters $\left(\tau_{c d s}, \tau_{s n}, \omega\right.$, and $\left.v_{s n}\right)$. With this remark, the proposed spectral model should be considered as a reasonable possibility in the absence of alternative models.

\section{CS INTERACTION AND DUSTY SHELL}

The interaction of the SN ejecta with the CSM is analysed using the hydrodynamic model in a thin shell approximation (Giuliani 1982; Chevalier 1982b). The central to the model is the equation of motion of the swept up thin shell driven by the dynamic pressure of SN ejecta $\rho_{S n}\left(R_{S} / t-v_{S}\right)^{2}$ and dynamic pressure of CSM $\rho_{c s}\left(v_{s}-v_{w}\right)^{2}$, where $R_{S}$ and $v_{s}$ are the radius and velocity of the thin shell, $v_{w}$ is the wind velocity taken to be $100 \mathrm{~km} \mathrm{~s}^{-1}$ (Fransson et al. 2014). The model has been described in details earlier (Chugai 2001), here we recap only key points. We do not distinguish between the swept up shells formed in the reverse and forward shocks: both are presumably merged into a single shell. The optical luminosity of the reverse and forward shocks at the age $t$ are calculated as the shock kinetic luminosities $0.5 \rho_{s n}\left(R_{s} / t-v_{s}\right)^{3}$ and $0.5 \rho_{c s}\left(v_{s}-v_{w}\right)^{3}$ multiplied by the radiation efficiency $\eta=t /\left(t+t_{c}\right)$ of a relevant shock, where $t_{c}$ is the cooling time. The radiation escape from both shocks is assumed to be instant, i.e., weak diffusion effects are ignored. The model does not include an additional luminosity related to the internal energy of an explosion.

The cooling time is calculated neglecting the lumpiness of the CSM and adopting postshock density to be equal to four-fold preshock density characteristic of a strong adiabatic shock with the adiabatic index $\gamma=5 / 3$. This approximation describes the energetically significant initial stage of the radiative shock cooling. The reverse shock in the considered case is always strongly radiative, while the forward shock is partially radiative with $\eta \sim 0.5$ between days 200 and 900. The lumpiness of the CSM could modify the cooling time of the forward shock. The effect however is not easy to estimate without detailed 3D-modelling. At first glance 
Table 2. Parameters of $\mathrm{H} \alpha$ models.

\begin{tabular}{cccccccccccc}
\hline Model & $v_{s n}$ & $v_{c d s}$ & $v_{c}$ & $v_{\max }$ & $q$ & $R_{2}$ & $\tau_{c d s}$ & $\tau_{s n}$ & $\omega$ & $f_{c d s}$ & $f_{s n}$ \\
\hline \multicolumn{1}{c}{$\mathrm{km} \mathrm{s}^{-1}$} \\
\hline $\mathrm{m} 172$ & 8500 & 5600 & 900 & 5300 & 0.9 & 1.13 & 0.07 & 0.58 & 0.6 & 0.05 & 0.3 \\
$\mathrm{~m} 400$ & 6100 & $(4700)$ & 1100 & 4400 & 0.6 & 1.1 & 0.14 & 1.15 & 0.2 & 0.05 & 0.12 \\
$\mathrm{~m} 510$ & 5700 & $(4200)$ & 850 & 3500 & 0.4 & 1.1 & 0.12 & 0.99 & 0.08 & 0.05 & 0.08 \\
$\mathrm{~m} 804$ & $(5000)$ & $(3200)$ & 850 & 3000 & 1.2 & 1.1 & 0.18 & 0.96 & 0.1 & $(0.02)$ & $(0.02)$ \\
\hline
\end{tabular}
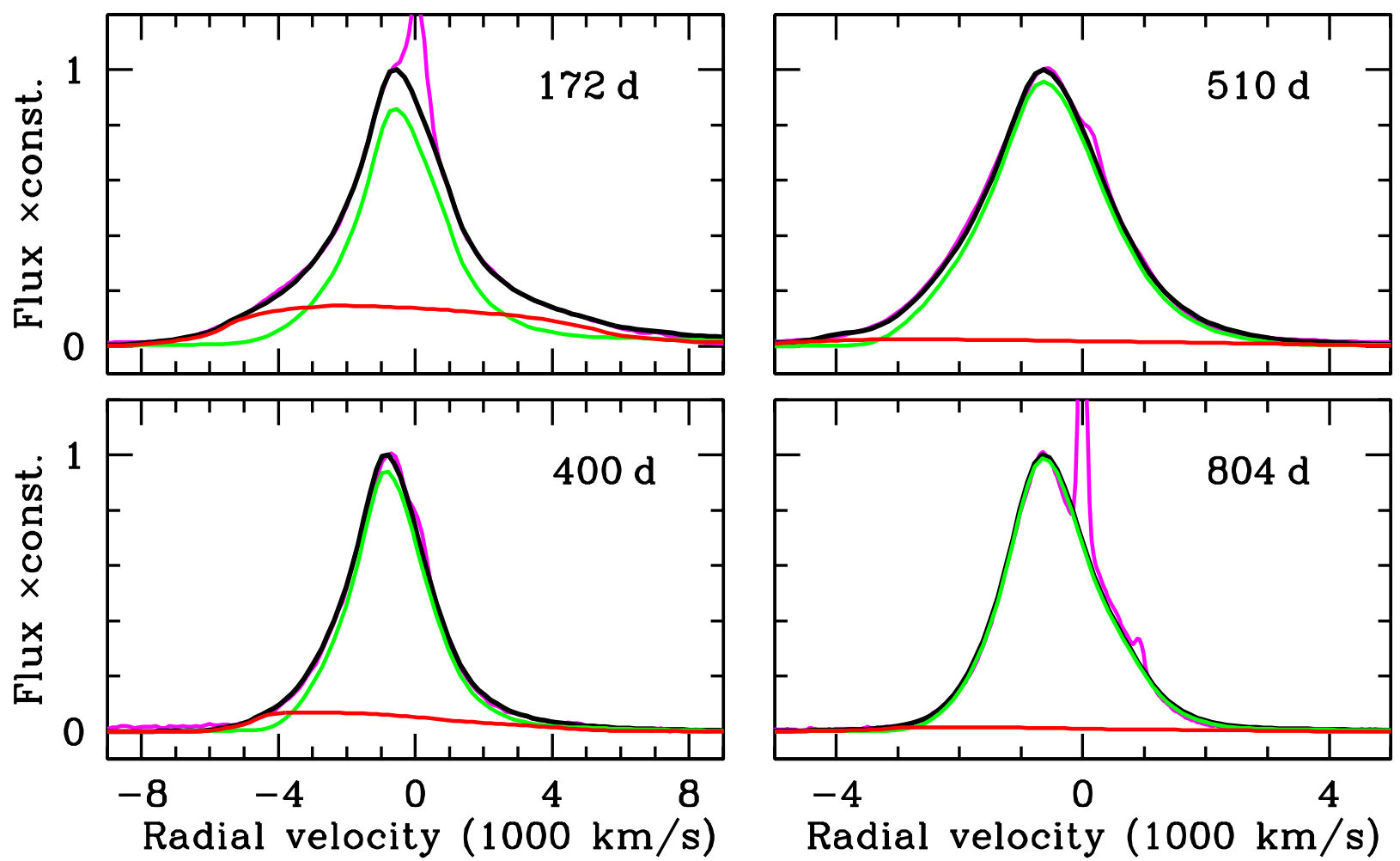

Figure 4. H $\alpha$ line in SN 2010jl (magenta) compared to the model profile at four epochs (black line). The blushifted profiles are well reproduced for adopted parameters (Table 2). The excess on day 172 around zero velocity is the narrow component, while on day 804 the excess is due to superimposed HII region. Shown are also contributions of the dominant intermediate component (green) and combined broad components of CDS and unshocked ejecta (red).

the presence of the dense shocked CS clouds should accelerate the radiative cooling. However, in a collision of forward shock rarefied flow and dense CS cloud most of the dissipated kinetic luminosity is deposited into the rarefied flow which results in the slowdown of the cooling process thus compensating effect of the dense shocked clouds. We therefore do not expect a significant modification of the bolometric light curve. As to the CDS dynamics, it is not affected by the cooling time modification.

The adopted density distribution of $\mathrm{SN}$ ejecta is $\rho=\rho_{0} /\left(1+\left(v / v_{0}\right)^{8}\right)$ with $\rho_{0}$ and $v_{0}$ determined by the ejecta mass and kinetic energy. The result is not very sensitive to the ejecta mass, nevertheless we consider two cases of ejecta mass: $M_{s n}=8 M_{\odot}$, the value used earlier for SN 2010j1 (Chandra et al. 2015), and $M_{s n}=30 M_{\odot}$. The remaining parameters are fixed by fitting to the bolometric light curve,
CDS, ejecta velocities (Table 2), and the CSM column density. The CSM density is set by a broken power low $\rho \propto r^{-s}$ with $s=0$ in the inner region $r<3.1 \times 10^{15} \mathrm{~cm}, s \sim 1.6 \ldots 2$ in the intermediate zone $r<4 \times 10^{16} \mathrm{~cm}$, and steep drop $(s=11)$ in the outer region.

The model with the ejecta mass of $8 M_{\odot}$ and kinetic energy of $E=3.9 \times 10^{51} \mathrm{erg}$ describes the observational bolometric luminosity, ejecta velocities, and CSM column density (Fig. 5). Note that the observational bolometric light curve (Fransson et al. 2014) is modified by the inclusion of the late $(t>460 \mathrm{~d})$ infrared luminosity reported by Fransson et al. (2014). The total mass of the CSM turns out to be $M_{C S}=3.9 M_{\odot}$. The model with $M_{s n}=30 M_{\odot}$ and $E=6.9 \times 10^{51} \mathrm{erg}$ (Fig. 6) describes data with the same CSM mass $M_{C S}=3.8 M_{\odot}$. Both models fit the data well and neither option is preferred. The main outcome of the modelling 

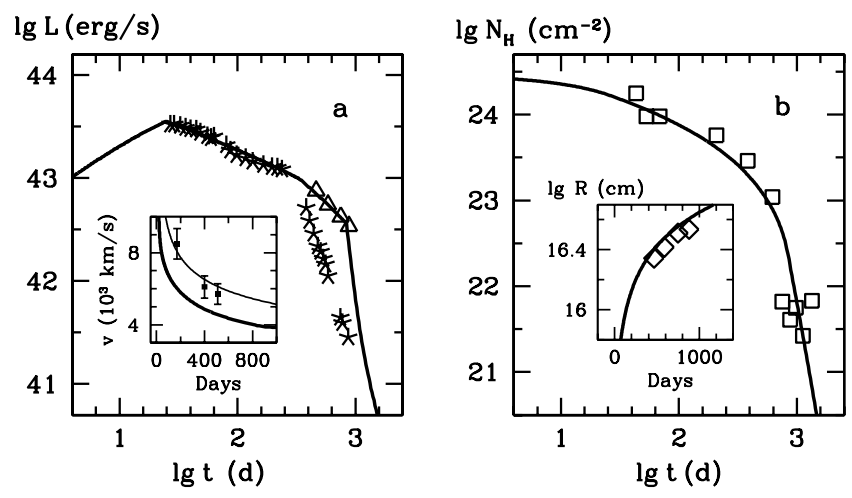

Figure 5. The SN/CSM interaction model for the ejecta mass of $8 M_{\odot}$ with observational data. Panel a shows the bolometric light curve compared to observational data from Fransson et al. (2014) (asterisks; IR luminosity summed with the optical is shown by triangles). Inset shows the boundary velocity of the unshocked ejecta (thin line) compared to data recovered from the $\mathrm{H} \alpha$ and the CDS velocity (thick line) Panel $\mathbf{b}$ shows the evolution of the CSM column density ahead of the forward shock compared to data (squares) (Chandra et al. 2015). Inset shows the CDS radius compared to the radius of the IR-emitting shell (diamonds) (Fransson et al. 2014).
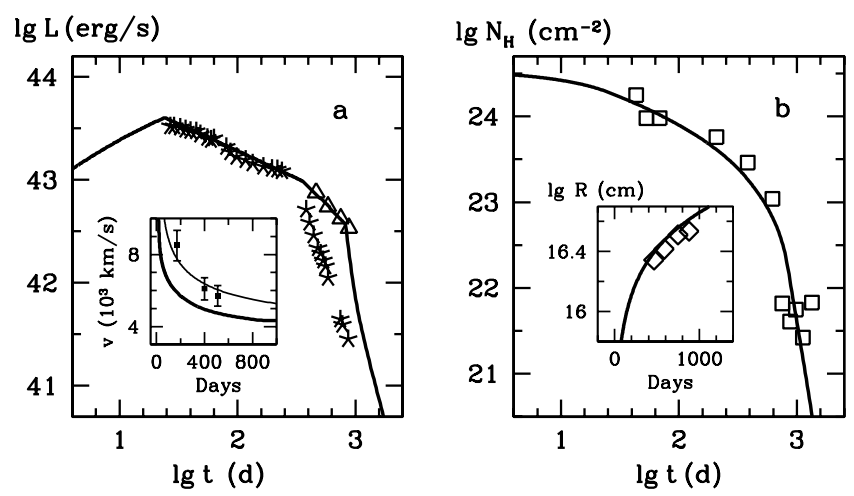

Figure 6. The same as Fig. 5 but for the ejecta mass of $30 M_{\odot}$

is the fact that in both models the CDS/SN radius at late time $t>460 \mathrm{~d}$ (Fig. 5, 6) coincides with the radius of the dusty shell reported by Fransson et al. (2014). This strongly suggests that the dust of CDS and/or unshocked ejecta is responsible for the late IR luminosity.

\section{DISCUSSION}

The aim of the paper has been to test a conjecture that in the late time SN 2010jl the dust forms in the CDS. To this end I propose a model explaining the Lorentz-like line profile as an outcome of hydrogen line emission from from shocked CS clumps in the forward shock. It is found that the macroscopic mixing of CS cloud fragments with the absorbing CDS and a significant absorption in the unshocked ejecta accounts for the observed blueshift. Noteworthy that the blueshift of hydrogen and He $1.083 \mu \mathrm{m}$ lines at the early stage $(<200$ d) may not be related with the dust absorption at all; the opacity is likely maintained by the excited hydrogen. The remarkable result suggested by the line profile modelling is that the absorption in the CDS is not sufficient: a significant absorption in the unshocked SN ejecta is needed to account for the whole blueshifted line profile. Moreover, the required optical depth of ejecta is 5-7 times larger compared to the CDS at the epoch 500-800 days. This indicates that the dust should form not only in the CDS but in the unshocked ejecta as well.

I found that the SN/CSM interaction model tuned by the fit to the bolometric light curve, ejecta velocities recovered from $\mathrm{H} \alpha$, and the CSM column density (Chandra et al. $2015)$ predicts the outer SN radius at late time $(t>460 \mathrm{~d})$ consistent with the radius of the dusty IR-emitting shell recovered by Fransson et al. (2014). The latter fact combined with the line blueshift analysis strongly suggests that at late time ( $>460 \mathrm{~d}$ ) the dust indeed forms both in the CDS and unshocked ejecta. Thus the conjecture of the dust formation in the CDS (Maeda et al. 2013; Gall et al. 2014) is only partially true: a significant amount of the dust should form in the unshocked ejecta. Moreover, the low covering factor of the CDS indicated by the small CDS optical depth $\sim 0.2$ at $510 \mathrm{~d}$ and $804 \mathrm{~d}$ suggests that the effective radius of the IR-emitting CDS photosphere turns out to be twice as small compared to the reported radius of the dusty shell (Fransson et al. 2014). This means that the late IR emission is likely primarily related to the more opaque dusty SN ejecta.

The radius of the late dust-emitting shell have been obtained (Fransson et al. 2014) using black body approximation, which suggests that the IR-emitting ejecta should be optically thick in the near IR band, e.g., at $3 \mu \mathrm{m}$ the optical depth must be $>1$. The direct consequence of that is the large optical depth at the $\mathrm{H} \alpha(\tau>10)$ assuming the absorption efficiency $Q_{a} \propto \lambda^{-1.5}$. This optical depth is by an order of magnitude larger than the value inferred from the $\mathrm{H} \alpha$ profile between days 500 and 800 (Table 2). The tension can be resolved assuming that the dust in ejecta is locked in opaque clumps, likewise in the model proposed for the dusty zone of SN 1987A ejecta (Lucy et al. 1991). Remarkably, the dust distribution in opically thick clumps for SN 2010jl has been already proposed by Maeda et al. (2013). In the case of clumpy dust distribution in unshocked SN ejecta the optical depth recovered from the blueshift assuming homogeneous model should be identified with the occultation optical depth $\tau_{o c}$, i.e., the number of clouds intersecting by the ejecta radius $R$.

One can estimate the amount of dust in SN ejecta adopting a simple model of opaque spherical dusty blobs of a radius $b$ homogeneously distributed in SN ejecta. The occultation optical depth is then $\tau_{o c}=\pi b^{2} n_{c} R=(3 / 4)(b / R)^{2} N_{c}$, where $n_{c}=3 N_{c} /\left(4 \pi R^{3}\right)$ is the number density of clouds and $N_{c}$ is the total number of clouds. The optical radius of the individual cloud is $\tau_{c}=n_{g}\left\langle\sigma_{a}\right\rangle b$, where $n_{g}$ is number density of grains, and $\left\langle\sigma_{a}\right\rangle$ is the absorption cross section of a grain averaged over the grain ensemble assuming grain radii distribution $d n / d a \propto a^{-3.5}\left(a_{\min }=3 \times 10^{-7} \mathrm{~cm}, a_{\max }=3 \times 10^{-5} \mathrm{~cm}\right)$ and using absorption efficiency of the carbon and silicate dust according to Draine \& Lee (1984). The total amount of the dust in SN ejecta is $M_{d}=(4 \pi / 3) b^{3} n_{c}\left\langle m_{g}\right\rangle N_{c}$, where $\left\langle m_{g}\right\rangle$ is the grain mass avaraged over the grain ensemble assuming the grain density $3 \mathrm{~g} \mathrm{~cm}^{-3}$. Expressing $N_{C}$ via $\tau_{o c}$ 
and $b$ via $\tau_{c}$ the dust mass thus reads

$M_{d}=(16 \pi / 9) R^{2} \tau_{c} \tau_{o c}\left\langle m_{g}\right\rangle /\left\langle\sigma_{a}\right\rangle$.

Inserting $\tau_{o c}=1$, the value derived from $\mathrm{H} \alpha$ on day 804, and the ejecta radius $R=3.8 \times 10^{16} \mathrm{~cm}$ obtained in the interaction model we infer from equation (2) the dust mass of $7.7 \times 10^{-4} \tau_{b} M_{\odot}$ for the graphite and $3.9 \times 10^{-3} \tau_{b} M_{\odot}$ for silicate dust. For optically thick blobs with $\tau_{b}=2$ at $3 \mu \mathrm{m}$ the amount of dust in ejecta is $8 \times 10^{-3} M_{\odot}$ for the silicate and $1.5 \times 10^{-3} M_{\odot}$ for the graphite dust. The conservative estimate of the dust amount is therefore $>10^{-3} M_{\odot}$.

In the IR data reported by Fransson et al. (2014) a signature of the hot dust becomes apparent only on day 465. In this regard, the blueshift at the early epoch (e.g. $172 \mathrm{~d}$ ) is unlikely related to the dust, rather it is due to the hydrogen opacity. To illustrate the possibility, consider the situation on day 172. Assuming the excitation temperature equal to the spectrophotometric estimate of $7500 \mathrm{~K}$ (cf. Fransson et al. 2014) and the CDS mass of $\approx 2 M_{\odot}$, according to the interaction model (Section 3), the optical depth due to the hydrogen continuum absorption at $6500 \AA$ assuming Saha-Boltzmann population distribution turns out to be $\sim 0.5$, sufficient to account for the CDS optical depth in the model m172. This consideration emphasises the point that the line blueshift in SNe IIn at early epoch cannot be a reliable argument in favour of the dust formation in CDS and/or ejecta unless supported by the blueshift emergence on the time scale $\ll t$.

The observed phenomena in late SN 2010jl, of course, are more complicated than our simplified consideration suggests. Particularly, we did not address the ratio of escaping optical-to-IR fluxes, implicitly assuming that the late IR luminosity is a result of the radiation cascade of the XUV radiation of the forward and reverse shocks. Meanwhile, at late time the optical-to-IR ratio is surprisingly small: around day 800 the difference is a factor of ten (Fransson et al. 2014). That low ratio cannot be explained in the picture in which the primary optical emission is comparable to the IR radiation and only the dust absorption is responsible for the low escaping optical luminosity. Indeed, despite the absorption, almost half of the emitted optical radiation could escape because of the small CDS optical depth $(\sim 0.2)$. The low optical-to-IR ratio at late time might be related to the conversion of the XUV radiation in the cool gas directly into the IR radiation avoiding significant emission of the optical radiation. This conjecture could be verified by the detection of strong IR features related to molecular species in supernovae of the similar category at the similar epoch (1.5-2 yr). An alternative possibility is that at late time the forward shock becomes adiabatic which accounts for the low optical luminosity while the high IR luminosity originates from the collisional heating of the newly formed dust mixed with the hot postshock gas.

Another puzzling issue is the weakness of the $\mathrm{H} \alpha$ broad boxy component related to the CDS material. A plausible answer can be seen from the following arguments. The strong upper limit for the $\mathrm{H} \alpha$ luminosity of a thin dense spherical shell with the radius $R$ in the case of very large line optical $\operatorname{depth}\left(\tau>10^{4}\right)$ is

$L(\mathrm{H} \alpha)=4 \pi^{2} R^{2} B_{v}(T) x_{e} \Delta v_{D}$,

where $B_{v}(T)$ is the black body intensity, $\Delta v_{D}$ is the ther- mal Doppler width for hydrogen, $x_{e}=(a \tau / \sqrt{\pi})^{1 / 3}$ is the local line width in units of the Doppler width in the case of the very opaque layer (Adams 1975), $a=3.3 \times 10^{-3}$ is the Voigt parameter for the $\mathrm{H} \alpha$ for $T \approx 7500 \mathrm{~K}$. For SN 2010jl on day 200 the shell radius is $R=1.4 \times 10^{16} \mathrm{~cm}$, and the CDS mass is $\approx 2 M_{\odot}$ (according to the interaction model in Section 3). Adopting the excitation temperature of $7500 \mathrm{~K}$ (Fransson et al. 2014), and assuming that the hydrogen is mostly neutral (which majorizes line width $x_{e}$ ) for solar hydrogen abundance one gets $L(\mathrm{H} \alpha)=0.9 \times 10^{41} \mathrm{erg} \mathrm{s}^{-1}$. Note that assumptions made above strongly overestimate the luminosity. Even in that case the obtained value is a factor of ten lower compared to the observed $\mathrm{H} \alpha$ luminosity of $\approx 10^{42}$ $\operatorname{erg} s^{-1}$ (Fransson et al. 2014) at that stage. If the CDS is not strongly disturbed, so that the area ratio for the cumulative surface $S$ of the line-emitting CDS material $A=S / 4 \pi R^{2} \sim 1$, then the broad boxy $\mathrm{H} \alpha$ related to the CDS turns out relatively weak $(<10 \%)$ and therefore gets overwhelmed by the broad wings of the ejecta component. The above argument have an interesting implication: the dominant contribution of the shocked CS clumps in the $\mathrm{H} \alpha$ luminosity suggests that the area ratio of the cumulative surface of the line-emitting CS cloud fragments should be large, of the order of $A \sim 10$.

\section{CONCLUSION}

I propose a simple model that accounts for the blueshifted line profiles in spectra of SN 2010jl at late time ( $\geq 500 \mathrm{~d})$. The key elements of this picture are (i) the shocked CS clouds in the forward shock, which are responsible for the dominant intermediate component of line profiles, (ii) the dusty cool dense shell with the small optical depth that accounts for the blueshift of the line maximum, and (iii) dusty unshocked SN ejecta that are responsible for the overall line blueshift. The modelling of the SN interaction with the CSM shows that the SN radius is consistent with the radius of optically thick dusty shell recovered from IR data. This taken together with the line profile analysis suggests that the dust formed in CDS and in unshocked SN ejecta is responsible for both the line blueshift and the IR emission. Most of the dust reside in optically thick clumps of the unshocked ejecta.

\section{ACKNOWLEDGEMENTS}

\section{REFERENCES}

Adams T. F., 1975, ApJ, 201, 350

Ambarzumian V. A., 1947, Doklady Acad. Sci. Armenia SSR, 6, 105 (in russian)

Andrews J. E., et al., 2010, ApJ, 715, 541

Andrews J. E., et al., 2011, AJ, 142, 45

Auer L. H., van Blerkom D., 1972, ApJ, 178, 175

Bevan A., Barlow M. J., 2016, MNRAS, 456, 1269

Blondin J. M., Ellison D. C., 2001, ApJ, 560, 244

Borish H. J., Huang C., Chevalier R. A., Breslauer B. M., Kingery A. M., Privon G. C., 2015, ApJ, 801, 7

Chandra P., Chevalier R. A., Irwin C. M., Chugai N., Fransson C., Soderberg A. M., 2012, ApJ, 750, L2

Chandra P., Chevalier R. A., Chugai N., Fransson C., Soderberg A. M., 2015, ApJ, 810, 32

Chevalier R. A., 1982a, ApJ, 258, 790

Chevalier R. A., 1982b, ApJ, 259, 302 
Chevalier R., Blondin J. M., 1995, ApJ, 444, 312

Chugai N. N., 1991, MNRAS, 250, 513

Chugai N. N., 2001, MNRAS, 326, 1448

Chugai N. N., 2009, MNRAS, 400, 866

Chugai N. N., Danziger I. J., 1994, MNRAS, 268, 173

Draine B. T., Lee H. M., 1984, ApJ, 285, 89

Dwek E., et al., 2017, ApJ, 847, 91

Elmhamdi A., et al., 2003, MNRAS, 338, 939

Filippenko A. V., 1991, in Woosley S. E., ed., Supernovae. p. 467

Fox O. D., Chevalier R. A., Dwek E., Skrutskie M. F., Sugerman B. E. K., Leisenring J. M., 2010, ApJ, 725, 1768

Fransson C., et al., 2014, ApJ, 797, 118

Gall C., et al., 2014, Nature, 511, 326

Giuliani Jr. J. L., 1982, ApJ, 256, 624

Hoffman J. L., Leonard D. C., Chornock R., Filippenko A. V., Barth A. J., Matheson T., 2008, ApJ, 688, 1186

Jencson J. E., Prieto J. L., Kochanek C. S., Shappee B. J., Stanek K. Z., Pogge R. W., 2016, MNRAS, 456, 2622

Klein R. I., McKee C. F., Colella P., 1994, ApJ, 420, 213

Klein R. I., Budil K. S., Perry T. S., Bach D. R., 2003, ApJ, 583,245

Lucy L. B., Danziger I. J., Gouiffes C., Bouchet P., 1989, in Tenorio-Tagle G., Moles M., Melnick J., eds, Lecture Notes in Physics, Berlin Springer Verlag Vol. 350, IAU Colloq. 120: Structure and Dynamics of the Interstellar Medium. p. 164, doi:10.1007/BFb0114861

Lucy L. B., Danziger I. J., Gouiffes C., Bouchet P., 1991, in Woosley S. E., ed., Supernovae. p. 82

Maeda K., et al., 2013, ApJ, 776, 5

Mattila S., et al., 2008, MNRAS, 389, 141

Ofek E. O., et al., 2014, ApJ, 781, 42

Osterbrock D. E., 1989, Astrophysics of gaseous nebulae and active galactic nuclei. University science books, Mill Valley, California

Pozzo M., Meikle W. P. S., Fassia A., Geballe T., Lundqvist P., Chugai N. N., Sollerman J., 2004, MNRAS, 352, 457

Sarangi A., Dwek E., Arendt R. G., 2018, preprint, (arXiv:1804.06878)

Smith N., Foley R. J., Filippenko A. V., 2008, ApJ, 680, 568

Smith N., et al., 2009, ApJ, 695, 1334

Smith N., Silverman J. M., Filippenko A. V., Cooper M. C., Matheson T., Bian F., Weiner B. J., Comerford J. M., 2012, AJ, 143,17

Stoll R., Prieto J. L., Stanek K. Z., Pogge R. W., Szczygieł D. M., Pojmański G., Antognini J., Yan H., 2011, ApJ, 730, 34

Utrobin V. P., Chugai N. N., 2015, A\&A, 575, A100

Yaron O., Gal-Yam A., 2012, PASP, 124, 668

Zhang T., et al., 2012, AJ, 144, 131 